\title{
64. Application of Microsurgical Technique for Intracranial Aneurysm Operation
}

\author{
Haruhiko Kikuchi, Hiroshi Matsumura, \\ Yasumasa Makita, Kitaro Kamata. \\ Kunio Nishikawa and Satoshi Kubo \\ Neurosurgical Department, Tenri Hospital
}

Since the biginning of 1970 we have had operations of intracranial aneurysms (54 cases, 65 aneurysms) by microsurgical method.

As to anterior communicating aneurysm, middle cerebral aneurysm, internal carotid aneurysm, we always use Yasargil's frontolateral sphenoidal approach.

Even on some case of basilar head aneurysm, we use this approach. Operation is performed on the condition of normothermia and normotension or hypotension with Arfonad. (about $70-80 \mathrm{mmHg}$ systolic for 5 to 40 minutes)

In general temporary clipping of parent vessels $(4 / 65)$ and blood transfusion during operation (9/54) are unnecessary with microsurgical technique.

With regard to multiple aneurysm, we made it a rule to treat all aneurysms on the same day even if bilateral craniotomy is required. (8 cases)

Sharp dissection under microscope brings prevention of premature rupture of aneurysm and circulatory disturbance of parent vessel.

Possibility of endoaneurysmorrhaphy is also a merit of microsurgery. In our series operative morbidity is $4.6 \%(3 / 65)$ and operative mortality is $3 \%(3 / 65)$.

\section{Microsurgey of Intracranial Aneurysms}

\author{
Isamu SAIto, Yuichi Ueda, Norihiko Basugi and Keiji SANo \\ Department of Neurosurgery, University of Tokyo
}

\section{Giant Intracranial Aneurysms}

Hideo Terao, Isao Muraoka and Korehito Kim Chikao Nagashima

Neurosurgical Service, Tokyo Hiroo Municipal Hospital

Masahisa Matsumoto

Department of Neurosurgery, Saitama Medical College

Neurosurgical Service, The National Railroad Central Hospital

$$
-165-
$$

